\title{
Sensitivity and Uncertainty Analysis by Discontinuous Galerkin of Lock-in Thermography for Crack Characterization
}

\author{
Ángel Javier Omella ${ }^{\mathrm{a}, \mathrm{b}, *}$, Ricardo Celorrio $^{\mathrm{c}}$, David Pardo ${ }^{\mathrm{a}, \mathrm{b}, \mathrm{d}}$ \\ ${ }^{a}$ Universidad del País Vasco (UPV/EHU), Leioa, Spain. \\ ${ }^{b}$ Basque Center for Applied Mathematics (BCAM), Bilbao, Spain. \\ ${ }^{c}$ Departamento de Matemática Aplicada, EINA/IUMA, Universidad de Zaragoza, Zaragoza, Spain. \\ ${ }^{d}$ Ikerbasque, Bilbao, Spain.
}

\begin{abstract}
This work focuses on the characterization of narrow vertical cracks of finite size using optically excited lock-in thermography (OLT). To characterize these cracks, we need to solve an ill-posed inverse problem. As a previous step to the solution of this inverse problem, we propose a sensitivity analysis to quantify the influence that the parameters involved in the model have on the surface temperature. Some of these parameters are estimated at the laboratory and they incorporate uncertainty that may severely affect the reconstruction of thin cracks. For this reason, we design a calibration criterion based on the sensitivity analysis to determine which parameters we need to include as unknowns of the inverse problem. We perform this analysis using a numerical discontinuous Galerkin method. Additionally, we propose a theoretical noise model for the thermograms. Then, we use a weighted least square method (WLS) to determine the parameters from the experimental thermograms. We also obtain a theoretical uncertainty of the reconstructed parameters in OLT-WLS fitting according to the used surface temperature dataset. Finally, we perform a numerical experiment with a $2.4 \mu \mathrm{m}$-thick vertical crack to show the sensitivities of the surface temperature with respect to the model parameters. We also determine the uncertainty of the parameters under different datasets with known noise characteristics.
\end{abstract}

Keywords: Crack characterization, Discontinuous Galerkin Method, Finite Element Method, Lock-in thermography, Sensitivity analysis

2010 MSC: 80M10, 80A23, 49Q12

\section{Introduction}

Lock-in thermography is an active technique used for nondestructive evaluation (NDE) of materials [1-4. It is based on the creation of a time-harmonic thermal wave [5, 6] in a sample and on the extraction of the amplitude and phase thermograms from its surface 5 by an infrared (IR) camera 7 .

\footnotetext{
* Corresponding author

Email address: ajaome@gmail.com (Ángel Javier Omella)

Preprint submitted to Comput. Methods Appl. Mech. Engrg.
} 
It is usual to classify the lock-in techniques based on the nature of the source that generates the thermal wave. The most relevant ones are: a) lock-in vibrothermography -also named ultrasound-excited lock-in thermography (ULT)-, in which stresses are generated in the sample by mechanical (ultrasound) excitation that results in a temperature 10 increase mainly on regions containing defects [8] 10]; b) induction lock-in thermography (ILT), in which an induction coil produces eddy currents in conductive materials, with the energy being dissipated as a modulated heat in the sample 11; and c) optically excited lock-in thermograpy (OLT), where the heat transfer is directly radiated on the material surface by using light coming from lasers or lamps 12 16. In this work, we 15 focus on OLT, which has demonstrated in the last decades the ability to detect small cracks 17 .

Nowadays, IR digital camera detectors incorporate one of the two following technologies: Charge-Coupled Devices (CCDs) or Complementary Metal Oxide Semiconductor (CMOS) [18. Both devices measure irradiation by counting the number of discrete inci-

20 dent photons into the sensor over the time of exposure. They then convert it into digital format, but the two technologies differ in how the conversion process is carried out. There is a plethora of works (see [19, 20, and references therein) devoted to describe the types of noises involved in the process of digital image generation, including photo response non-uniformity (multiplicative type noise), photon noise (Poisson type noise), thermal ${ }_{25}$ dark current noise, read noise, and quantization noise (additive type noise). The prevalence of each noise type differs depending on the technology of the IR camera detector but the noises origins are essentially the same in both cases.

Continuous models of heat diffusion in a cracked media require additional domains to model the air layer existing in the gap formed by the crack [21. When a traditional 30 continuous model is solved by a finite element method, it is necessary to mesh the gap, dramatically increasing the problem size. This leads to a prohibitive computational cost for problems involving micro-cracks. To overcome this problem, in 22] we proposed a model with a discontinuity in temperature. In there, thin cracks were modeled as interfaces characterized by their thermal resistances inside a homogeneous bulk. To 35 solve the problem, we applied a Discontinuous Galerkin (DG) method (see [17, 23, 24).

Despite the above advances on the modeling of cracks, an efficient quantitative analysis for micro-cracks characterization still needs to be established. Here, we address this challenge from different points of view.

We first realize that some of the parameters involved in the direct problem are es-

40 timated at the laboratory and they contain uncertainty. If we consider a modulated and focused laser beam as the source that generates the time-harmonic heat flux on the sample, such estimated parameters are the radius of the laser beam and its center. To quantify the influence in the surface temperature produced by a small variation in the parameters, we perform a sensitivity analysis. In addition, we propose a calibration cri-

45 terion to determine the parameters estimated at the laboratory that should be included as unknowns in the inverse problem to characterize the crack with a fixed tolerance.

We additionally notice that the experimental data (amplitude and phase thermograms) recorded by the IR camera are noisy. Thus, we propose a theoretical noise model for the data based on physical considerations about noise sources in image acquisition.

${ }_{50}$ This leads us to propose general weighted least square (WLS) fitting methods for the complex-valued version of the thermograms. We analyze three extreme cases where one type of noise is dominant over the others: the fitting of the thermogram (when additive 
noise is dominant), the fitting of the logarithm of the thermogram (when multiplicative noise is dominant), and the fitting of the result of applying the square root to the

55 thermogram amplitude (when Poisson-type noise is dominant). We also show how the uncertainty of the complex-valued thermograms is transmitted to the real-valued unknowns included in the inverse problem. This uncertainty depends on the data subset selected to perform the WLS fitting.

The remainder of the manuscript is organized as follows. Section 2 recalls the discontinuous model for steady-state thermal waves in the presence of cracks and the DG numerical method we select to solve it. Section 3 shows the numerical results in an OLT inspection of a sample with a crack of thickness $2.4 \mu \mathrm{m}$. It next introduces the complex-valued sensitivity and shows the numerical sensitivities. Then, Section 4 describes the procedure to apply the calibration criterion over the parameters estimated

65 at the laboratory. Section 5 proposes a simplified model of lock-in image including the noise. After that, Section 6 introduces WLS fitting method and illustrates its behavior with the three extreme noise-type cases. We also provide a theoretical uncertainty map of the reconstructed parameters in OLT least square fitting. The section ends describing the influence of dataset sampling on the uncertainty determination of the parameters.

70 The last section is devoted to conclusions.

\section{Modeling and Numerical Method}

We consider a cracked domain $\Omega$ (see Figure 1), which contains a homogeneous, opaque, and isotropic solid sample with thermal conductivity $\kappa$ and thermal diffusivity $\alpha$. The crack placed at the interface $\Gamma_{c}$ is characterized by its thermal resistance $R: \Gamma_{c} \rightarrow$

$75 \mathbb{R}^{+}$. The effective thickness of the crack is $R \kappa_{a}$, where $\kappa_{a}$ is the thermal conductivity of the fluid (usually air) that resides inside the crack. A laser beam incides on the domain boundary illuminating part of it. We define two zones: the illuminated zone denoted as $\Gamma_{I}$ and the shadowed zone $\Gamma_{S}$, so $\partial \Omega=\Gamma_{I} \cup \Gamma_{S}$. We denote as $\boldsymbol{x}_{\boldsymbol{c}} \in \Gamma_{I}$ the point where the center of the laser beam incises. We assume that the normal vector at this

so point, denoted as $\boldsymbol{n}_{\boldsymbol{x}_{\boldsymbol{c}}}$, is parallel to the direction of the incident laser beam. Notice that the normal vector $\boldsymbol{n}_{\boldsymbol{x}}$ of an arbitrary point $\boldsymbol{x} \in \Gamma_{I}$ does not have to be parallel to the incident laser beam direction (see Figure 1).

We introduce the following jump $\llbracket \cdot \rrbracket$ and average $\{\cdot\}$ trace operators defined on any possible interior interface $\Gamma \subset \bar{\Omega}$,

$$
\llbracket \cdot \rrbracket:=(\cdot)^{+} \boldsymbol{n}_{+}+(\cdot)^{-} \boldsymbol{n}_{-} \quad\{\cdot\}:=\frac{(\cdot)^{+}+(\cdot)^{-}}{2},
$$

where $\boldsymbol{n}_{+}$and $\boldsymbol{n}_{-}$are the outward normal vectors to each side of the interface $\Gamma$, and $(\cdot)^{+}$and $(\cdot)^{-}$are the lateral limit operators of the scalar or vector valued functions at 85 each side of the interface.

Time harmonic sources operating at frequency $\nu$ increase the temperature on $\Omega$. Once the quasi-steady state is reached, the temperature in the sample is a superposition of the room temperature, a stationary temperature, and a harmonic temperature. This harmonic state is the so-called thermal wave, whose mathematical representation is:

$$
\theta(\boldsymbol{x}, t)=\Re\left(u(\boldsymbol{x}) e^{-i 2 \pi \nu t}\right) \quad \text { in } \Omega .
$$




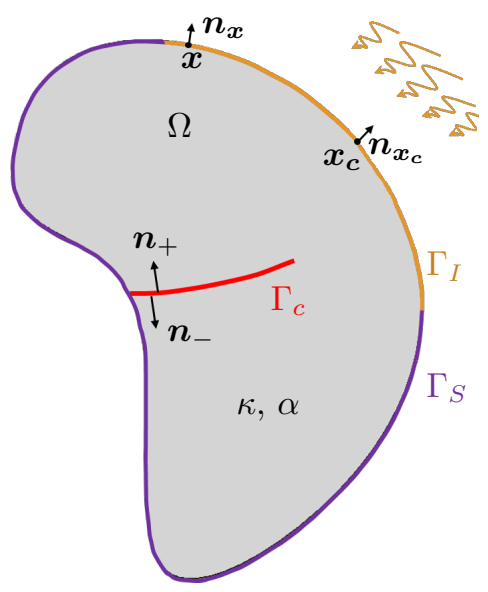

Figure 1: Scketch of a cracked domain. The crack is modelled as an interface $\Gamma_{c}$ with thermal resistance $R$. The illuminated part of the boundary is denoted as $\Gamma_{I}$, and the shadowed one as $\Gamma_{S}$.

The spatial component - phasor or complex amplitude- of the thermal wave, $u: \Omega \rightarrow \mathbb{C}$, is governed by the following discontinuous transmission problem:

$$
\left\{\begin{array}{rlrl}
\Delta u+i \frac{2 \pi \nu}{\alpha} u & =f_{1}, \\
\llbracket \kappa \nabla u \rrbracket & =f_{2}, & & \text { in } \Omega \backslash \Gamma_{c}, \\
\llbracket u \rrbracket+R\{(\kappa \nabla u \cdot \boldsymbol{n}) \boldsymbol{n}\} & =\boldsymbol{f}_{\mathbf{3}}, & & \text { on } \Gamma_{c}, \\
\kappa \nabla u \cdot \boldsymbol{n} & =g, & & \text { on } \Gamma_{c},
\end{array}\right.
$$

where $-\kappa f_{1}$ is the spatial component of a volumetric time-harmonic heat source, $f_{2}$ appears in models of vibrothermography and is the complex amplitude due to a periodic heat flux induced on the crack, $f_{3}$ is the spatial component of a time-harmonic thermal dipole oriented in a perpendicular direction to $\Gamma_{c}$, and $g$ is the complex amplitude of the time-harmonic heat flux acting on the boundary.

In an OLT with opaque samples, we consider $f_{1}=f_{2}=0$, and $\boldsymbol{f}_{\mathbf{3}}=\mathbf{0}$. We model the source $g$ as a Gaussian type function on the illuminated zone of the boundary, assuming that the laser beam is perpendicular to the boundary at point $\boldsymbol{x}_{\boldsymbol{c}}$ :

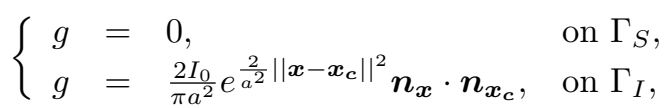

where $I_{0}$ is a parameter that indicates the intensity and the phase of the illumination, $a$ 95 is the radius of the circular spot centered at point $\boldsymbol{x}_{\boldsymbol{c}}=\left[x_{c 1}, x_{c 2}, x_{c 3}\right]^{T}$, and $\boldsymbol{n}_{\boldsymbol{x}}, \boldsymbol{n}_{\boldsymbol{x}_{\boldsymbol{c}}}$ are the outward normal vectors at points $\boldsymbol{x}$ and $\boldsymbol{x}_{\boldsymbol{c}}$, respectively.

The DG discretization proposed to solve (3) is performed according to 23 . For that, we first define $\Omega_{h}$ and $\Gamma_{c, h}$ as polygonal approximations of the given domain $\Omega$ and interface $\Gamma_{c}$, respectively. We select a discretization $\Omega_{h}$ conforming to $\Gamma_{c, h}$ by means of a triangulation $\mathcal{T}_{h}:=\left\{K_{j}\right\}_{j=1}^{M}$ of simplexes $K_{j}$. We denote by $\Gamma_{s k}$ to the skeleton of the triangulation without the facets that belong to the cracks or to the boundary, i.e., $\Gamma_{s k}:=$ 
$\left(\bigcup_{j=1}^{M} \partial K_{j}\right) \backslash\left(\Gamma_{c, h} \cup \partial \Omega_{h}\right)$. Then, we define the finite dimensional complex-valued space $V_{h}:=\left\{v_{h}: \Omega_{h} \rightarrow \mathbb{C}\left|v_{h} \in L^{2}, v_{h}\right|_{K} \in \mathcal{P}_{p}(K), \forall K \in \mathcal{T}_{h}\right\}$, where $\mathcal{P}_{p}(K)$ is the space of complex-valued polynomials of degree $p \geq 2$. Finally, we compute the approximated solution $u_{h}$ by solving the following DG formulation (see [22] for its derivation):

Find $u_{h} \in V_{h}$ such that:

$$
\begin{array}{ll}
\sum_{K \in \mathcal{T}_{h}} \int_{K} \nabla u_{h} \cdot \nabla v_{h}-i \frac{2 \pi \nu}{\alpha} \int_{\Omega_{h}} u_{h} v_{h}+\int_{\Gamma_{s k} \cup \Gamma_{c, h}} \llbracket u_{h} \rrbracket \cdot\left\{\nabla v_{h}\right\}-\int_{\Gamma_{s k} \cup \Gamma_{c, h}}\left\{\nabla u_{h}\right\} \cdot \llbracket v_{h} \rrbracket \\
\quad+\int_{\Gamma_{c, h}} \kappa R\left\{\nabla u_{h} \cdot \boldsymbol{n}\right\}\left\{\nabla v_{h} \cdot \boldsymbol{n}\right\} & \\
=-\int_{\Omega_{h}} f_{1} v_{h}+\frac{1}{\kappa} \int_{\partial \Omega_{h}} f_{2}\left\{v_{h}\right\}+\int_{\Gamma_{c, h}} f_{3} \cdot\left\{\nabla v_{h}\right\}+\frac{1}{\kappa} \int_{\partial \Omega_{h}}^{g} v_{h}, & \text { for all } v_{h} \in V_{h} .
\end{array}
$$

\section{Simulation in OLT Inspection of a Micro-cracked Material and Sensitivity Analysis}

The solution of (5) provides the complex temperature field in the entire domain for a given source. However, some parameters (the radius $a$, and the center $\boldsymbol{x}_{\boldsymbol{c}}$ ) of the source $g$ defined in (4) are only known with pixel resolution, which may be insufficient. In addition, the diffusivity $\alpha$ may incorporate some error. The uncertainty of these parameters can severely affect the accuracy to recover $R$ by OLT techniques.

To overcome the above problem, in this section we analyze the sensitivity of the temperature on the surface where the experimental thermograms are extracted, with respect to $a, \boldsymbol{x}_{\boldsymbol{c}}$, and $\alpha$ together with the thermal resistance. To numerically illustrate these sensitivities, we propose a specific OLT experiment composed of a bulk with a finite vertical thin crack (the least sensitive case) excited by a focused laser beam. All DG implementations have been performed in the free software FEniCS [25].

\subsection{Numerical Experiment Description and Thermograms Representation}

We consider an OLT experiment (see Figure 2 ) at frequency $\nu=1 \mathrm{~Hz}$ over a sample with AISI-304 stainless steel thermal properties $\left(\kappa=15 \mathrm{Wm}^{-1} s^{-1}\right.$ and $\alpha=4$. $10^{-6} \mathrm{~m}^{2} \mathrm{~s}^{-1}$ ). The superficial vertical crack, filled with air, has a size equal to $1 \mathrm{~mm} \mathrm{x}$ $1 \mathrm{~mm} \times 2.4 \mu \mathrm{m}$ (length, depth, and thickness, respectively). This provides a thermal resis-

115 tance of $R=10^{-4} \mathrm{~m}^{2} \mathrm{KW}^{-1}$. It is placed in the perpendicular direction to the illuminated surface (plane $x_{3}=0$ ), along the axis $x_{1}$, and centered at the origin. The source $g$ is modeled according to $(4)$, with $a=7.5 \cdot 10^{-4} \mathrm{~m}$, and $\boldsymbol{x}_{\boldsymbol{c}}=[0,1.5,0]^{T} \cdot 10^{-3} \mathrm{~m}$.

Figure 3 shows the computed normalized temperature on the illuminated surface $\left(x_{3}=0\right)$, the normalized amplitude thermogram $\left|u_{N}\right|(3 \mathrm{a})$, and the phase $\phi\left(u_{N}\right) 3 \mathrm{~b}$.

${ }_{120}$ Thermograms $(3 \mathrm{C})$ and $(3 \mathrm{~d})$ exhibit a discontinuity on the solution along the crack.

\subsection{Complex-valued Sensitivity}

The sensitivity $S_{s}: \Omega \rightarrow \mathbb{C}$ of the solution from 3 with respect to a parameter $s \in \mathbb{R} \backslash\{0\}$ allows us to quantify the influence that a perturbation of the parameter $s$ 

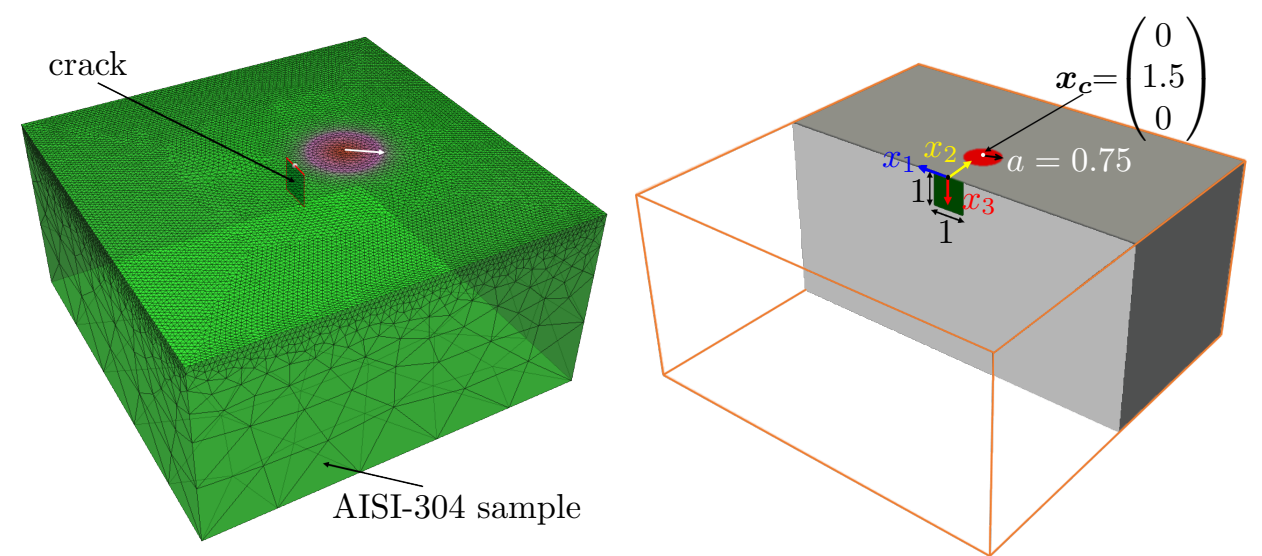

Figure 2: Mesh and OLT simulation sketch. Units are expresed in milimeters. The green rectangle represents a crack with $R=10^{-4} \mathrm{~m}^{2} \mathrm{KW}^{-1}$.

(i.e. $x_{c 1}, x_{c 2}, a, \alpha$, and $R$ ) has in the solution. We define it as:

$$
S_{s}:=\frac{\partial \log u}{\partial \log s}=\frac{s}{u} \frac{\partial u}{\partial s} .
$$

As indicated in 26, it is possible to perform a physical interpretation of the real and imaginary parts of the sensitivity according to the equation

$$
\log u=\log |u|+i \phi_{u}
$$

where $\phi_{u}$ is the phase of $u$. The real part of the sensitivity $S_{s}$ is related with relative changes in the temperature amplitude $8 \mathrm{a}$. Phase variations are related to the imaginary part of the sensitivity $8 \mathrm{~b}$. Mathematically,

$$
\begin{array}{r}
\Re\left(S_{s}\right)=\frac{\partial \log |u|}{\partial \log s}=s \frac{\partial \log |u|}{\partial s}, \\
\Im\left(S_{s}\right)=\frac{\partial \phi_{u}}{\partial \log s}=s \frac{\partial \phi_{u}}{\partial s} .
\end{array}
$$

In order to compare the magnitudes of the sensitivity of the solution with respect to the parameters of the model, we express these sensitivities through its module $\left|S_{s}\right|$.

To compute the sensitivities associated to the experiment showed in subsection 3.1. we first estimate the partial derivatives of the temperature with respect to each parameter $s$. The strong formulation associated to each partial derivative is found by solving (3) after replacing the following terms:

- $u$ by $u_{x_{c 1}}$, where $u_{x_{c 1}}:=\frac{\partial u(x)}{\partial x_{c 1}}$; with the sources $g=\frac{8 I_{0}\left(x_{1}-x_{c 1}\right)}{\pi a^{4}} e^{-\frac{2}{a^{2}}\left\|\boldsymbol{x}-\boldsymbol{x}_{c}\right\|^{2}}$, $f_{1}=f_{2}=0$, and $\boldsymbol{f}_{\mathbf{3}}=\mathbf{0}$.

- $u$ by $u_{x_{c 2}}$, where $u_{x_{c 2}}:=\frac{\partial u(x)}{\partial x_{c 2}}$; with the sources $g=\frac{8 I_{0}\left(x_{2}-x_{c 2}\right)}{\pi a^{4}} e^{-\frac{2}{a^{2}}\left\|\boldsymbol{x}-\boldsymbol{x}_{c}\right\|^{2}}$, $f_{1}=f_{2}=0$, and $\boldsymbol{f}_{\mathbf{3}}=\mathbf{0}$. 


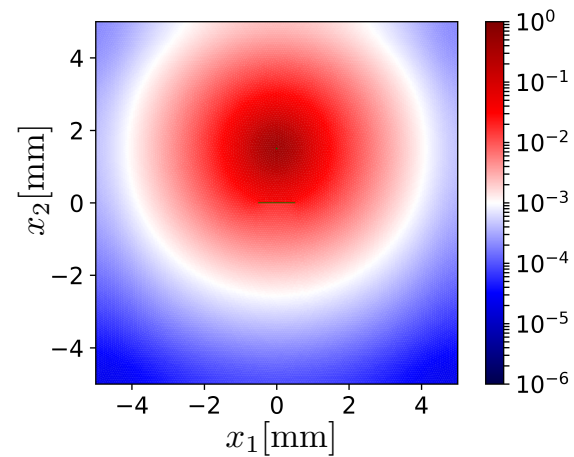

(a) Normalized amplitude thermogram.

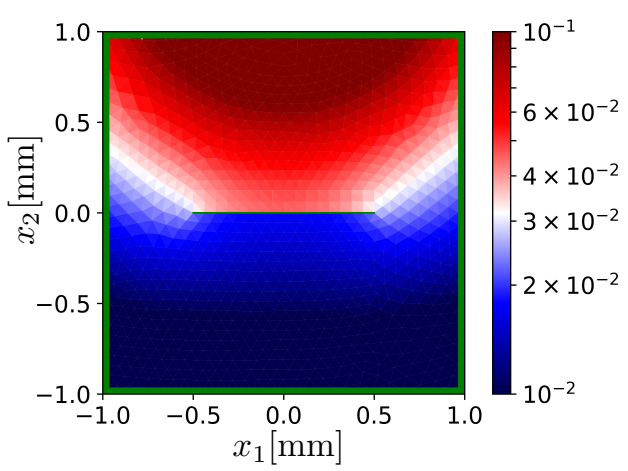

(c) Detail of normalized amplitude thermogram.

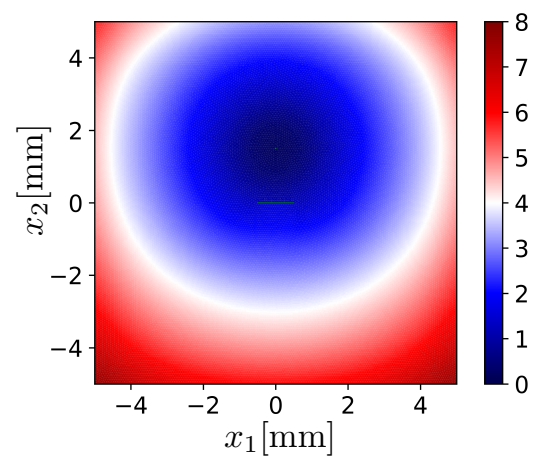

(b) Normalized phase thermogram (in radians).

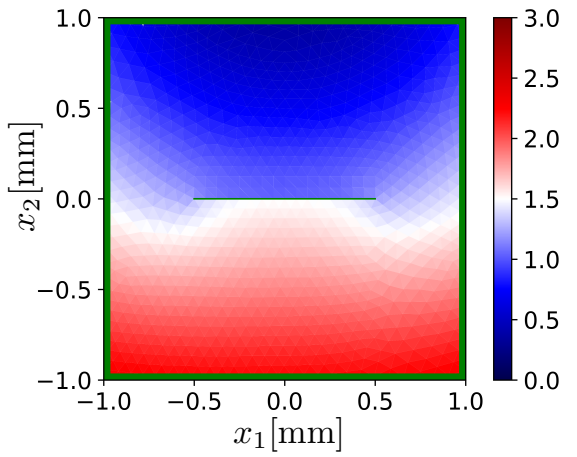

(d) Detail of normalized phase thermogram (in radians).

Figure 3: Normalized solution $u_{h}$ in the illuminated plane $\left(x_{3}=0\right)$ and around the crak. The green line depicts the location of the crack.

- $u$ by $u_{a}$, where $u_{a}:=\frac{\partial u(x)}{\partial a}$; with the sources $g=\frac{4 I_{0}\left(2|| \boldsymbol{x}-\boldsymbol{x}_{\boldsymbol{c}} \|^{2}-a^{2}\right)}{\pi a^{5}} e^{-\frac{2}{a^{2}}\left\|\boldsymbol{x}-\boldsymbol{x}_{c}\right\|^{2}}$, $f_{1}=f_{2}=0$, and $\boldsymbol{f}_{\mathbf{3}}=\mathbf{0}$.

- $u$ by $u_{R}$, where $u_{R}:=\frac{\partial u(x)}{\partial R}$; with the sources $f_{1}=f_{2}=g=0$ and $\boldsymbol{f}_{\mathbf{3}}=$ $-\{\kappa(\nabla u \cdot \boldsymbol{n}) \boldsymbol{n}\}$.

- $u$ by $u_{\alpha}$, where $u_{\alpha}:=\frac{\partial u(x)}{\partial \alpha}$; with the sources $f_{1}=i \frac{2 \pi \nu}{\alpha^{2}} u, f_{2}=g=0$, and $\boldsymbol{f}_{\mathbf{3}}=\mathbf{0}$.

Once each partial derivative is implicitly defined, we solve the corresponding problems using a DG method (5). Finally, we apply (6) to obtain the complex sensitivity $S_{s}$ associated to each parameter.

\subsection{Numerical Sensitivities}

Figure 4 shows the module of sensitivity $\left|S_{s}\right|$ on the illuminated plane $\left(x_{3}=0\right)$ for all the parameters under study $s \in\left\{x_{c 1}, x_{c 2}, a, \alpha, R\right\}$. These sensitivities are discontinuous 
along the crack. Moreover, they seem to be linearly independent. Therefore, there is a hope to properly invert all of them simultaneously. We also note that $S_{R}$ has quasi-local support around the crack. Within this area, other parameters have larger sensitivity values than $S_{R}$. This indicates that the thermal resistance can only be accurately approximated if the remaining parameters are determined with high accuracy.

\section{Calibration Criterion}

In the practical resolution of inverse problems, we minimize the discrepancy between the measurements and the solution of the direct problem. An inverse problem has its own unknowns in addition to other parameters that have been estimated in the laboratory with measurement errors. The uncertainty in the solution of the inverse problem generated by a measurement error depends on the sensitivity associated to the measured parameter: the higher the sensitivity the larger the uncertainty. If the induced uncer155 tainty is lager than a given threshold, the corresponding parameter should be considered as an unknown of the inverse problem. In our case, the model parameters related to the source $\left(a, x_{c_{x 1}}\right.$, and $\left.x_{c_{x 2}}\right)$ are given from laboratory experiments with pixel resolution. Thus, they are inexact. The value attributed to $\alpha$ incorporates uncertainty too. Let us denote by $r$ to the set of parameters determined with measurement errors, namely, ${ }_{160} r=\left\{x_{c_{x 1}}, x_{c_{x 2}}, a, \alpha\right\}$. This set of parameters influences the recovery of the thermal resistance $R$ by means of the solution of the inverse problem.

We propose a calibration criterion that determines which a priori known parameters we need to incorporate in the inverse problem in order to be able to determine $R$ with a given tolerance.

\subsection{Calibration Criterion Algorithm}

The calibration criterion is as follows:

1. Compute the sensitivities modulus of each parameter applying (6) at each side of the interface $\Gamma_{c}$ in the illuminated plane. We denote by $\left|S_{s}^{+}\right|$and $\left|S_{s}^{-}\right|$to the sensitivities modulus of the parameter $s$ at the side near and far from the illumination center $\boldsymbol{x}_{c}$, respectively.

2. Calculate the average of the sensitivities modulus

$$
\overline{\left|S_{s}\right|}:=\int_{\Gamma_{C} \cap \partial \Omega}\left\{\left|S_{s}\right|\right\} d x .
$$

3. Fix the acceptable relative error standard deviation (tolerance) of $R$, denoted as $\sigma_{\frac{\Delta R}{R}}$.

4. Estimate the standard deviation of the relative error for each parameter $\sigma_{\frac{\Delta}{|r|}}$.

5. Verify compliance or not with the calibration criterion for each parameter in $r$ :

$$
\sigma_{\frac{\Delta r}{|r|}}<c \frac{\overline{\left|S_{R}\right|}}{\overline{\left|S_{r}\right|}} \sigma_{\frac{\Delta R}{R}}
$$




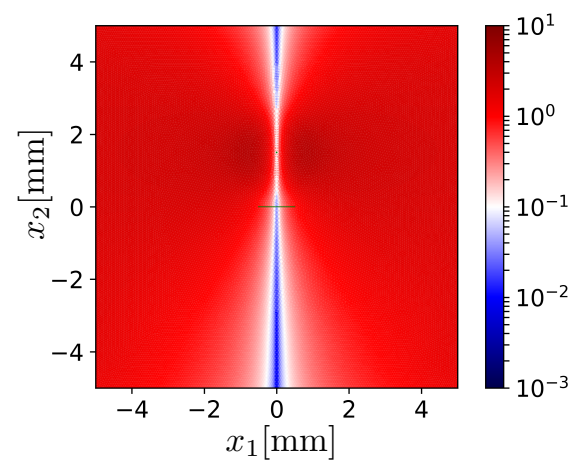

(a) $\left|S_{x_{c 1}}\right|$

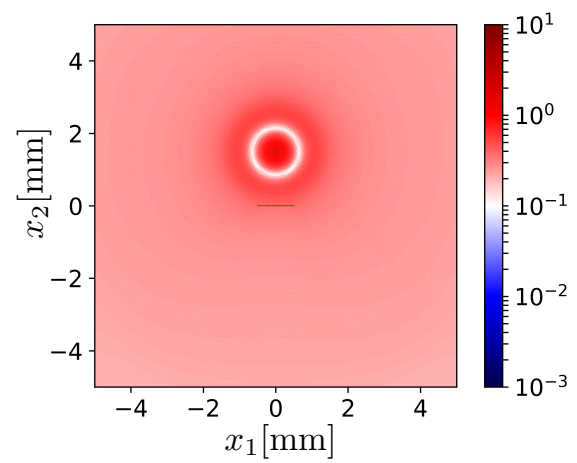

(c) $\left|S_{a}\right|$

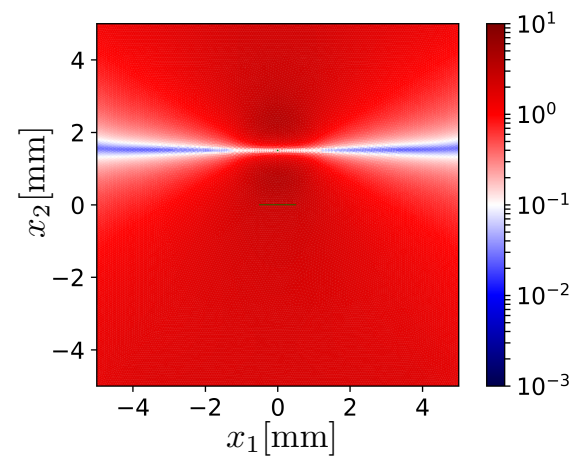

(b) $\left|S_{x_{c 2}}\right|$

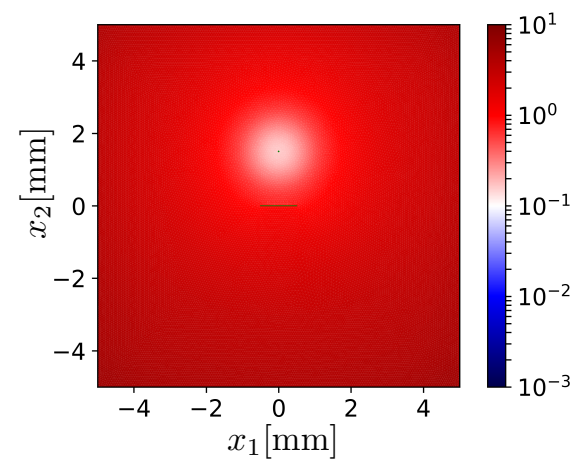

(d) $\left|S_{\alpha}\right|$

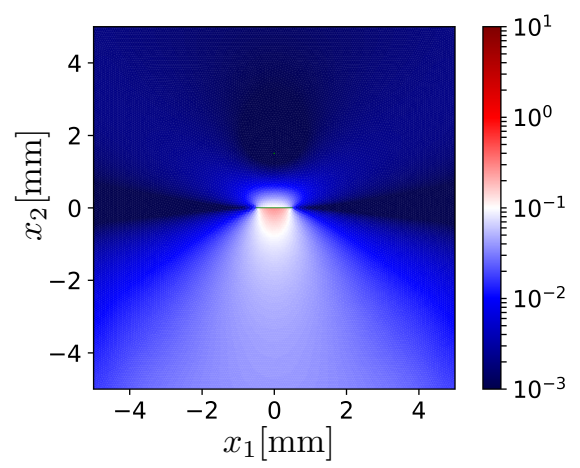

(e) $\left|S_{R}\right|$

Figure 4: Sensitivities on the illuminated plane $\left(x_{3}=0\right)$. The green line and point depict the crack and the location of the illumination center, respectively.

where $c$ is a constant to control the weight with which we impose the criterion. We select $175 \quad c=0.1$.

If condition 10 is satisfied, we refrain from including parameter $r$ as a new unknown 
of the inverse problem. Otherwise, the parameter will be incorporated in the inverse problem as an unknown initialized with the value determined at the laboratory.

\subsection{Numerical Application of the Calibration Criterion}

180

We apply the calibration criterion in the experiment described in Section 3.1 to determine the parameters that will be included as unknowns of the inverse problem.

Figure (5) shows the results of $\left|S_{s}^{+}\right|,\left|S_{s}^{-}\right|$, and $\mid \overline{S_{s} \mid}$. Then, we fix the assumed relative error to determine the thermal resistance in the range of $25 \%$, that is, $\sigma_{\frac{\triangle R}{R}}=0.25$.

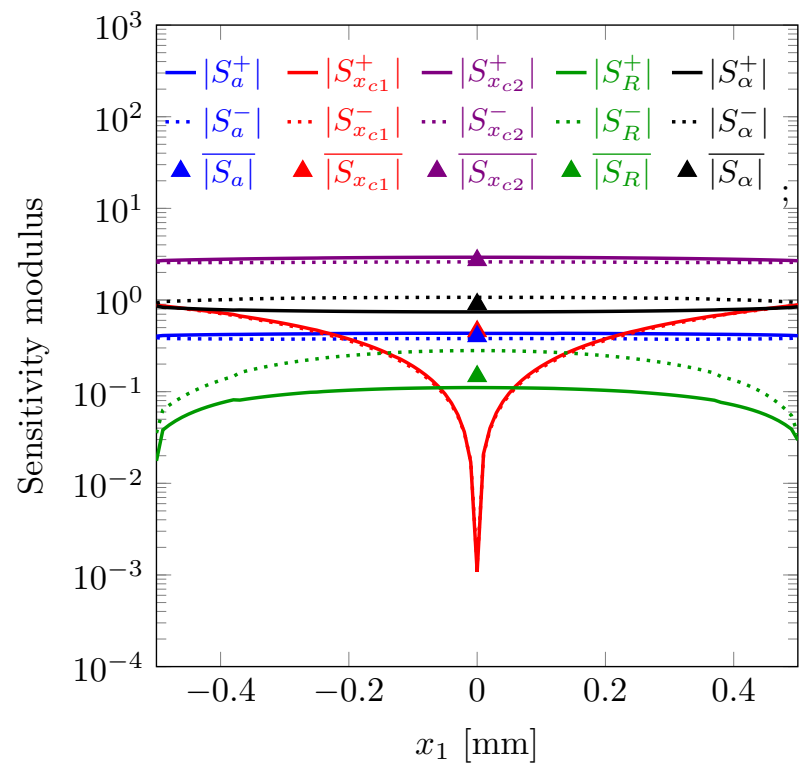

Figure 5: Sensitivity modulus of the parameters along the crack in the illuminated plane $\left(x_{3}=0\right)$. Each color represents one parameter $s \in\left\{a, x_{c 1}, x_{c 2}, R, \alpha\right\}$. The continuous line represents the values in the crack-side near to the source whereas the dotted line represents the far away ones. Each triangle depicts the average sensitivity modulus along the crack.

We then need to estimate the relative errors of the remaining parameters via laboratory experiments. We assume that the spatial resolution of the grid projected on the surface sample by the detector cells of the IR camera in an OLT experiment is $30 \mu \mathrm{m}$ (see [12]). We estimate the uncertainty associated with the laser spot center location at each direction to be approximately $45 \mu \mathrm{m}$. As $\left\|\boldsymbol{x}_{c}\right\|=1500 \mu \mathrm{m}$, then $\sigma_{\frac{\Delta x_{c}}{\left\|\boldsymbol{x}_{c}\right\|}} \approx \sigma_{\frac{\Delta x_{c 2} \|}{\left\|\boldsymbol{x}_{c}\right\|}} \approx 3 \cdot 10^{-2}$. For the radius, we estimate the error to be also $45 \mu \mathrm{m}$, which corresponds to a relative error of $\sigma_{\frac{\Delta a}{a}} \approx 6 \cdot 10^{-2}$. In here, we assume for simplicity that the relative error for the thermal diffusivity of the sample made in AISI-304 is close to $0.1 \%$, although in practice, this relative error may be higher [27.

Finally, we evaluate the compliance of the calibration criterion 100. Table 1 shows this evaluation over each parameter determined at the laboratory. We observe that $\alpha$ 195 is the only parameter that satisfies the imposed requirement to achieve the assumed $25 \%$ percentage error in the recovery of the thermal resistance. Therefore, it may not 
Table 1: Evaluation of compliance with the calibration criterion for each parameter

\begin{tabular}{ccc}
\hline Parameter & Calibration criterion & Compliance \\
\hline$x_{c_{x 1}}$ & $3 \cdot 10^{-2} \nless 0.1 \frac{0.145}{0.46} 0.25 \lesssim 7.9 \cdot 10^{-3}$ & No \\
$x_{c_{x 2}}$ & $3 \cdot 10^{-2} \nless 0.1 \frac{0.145}{2.7} 0.25 \lesssim 1.35 \cdot 10^{-3}$ & No \\
$a$ & $6 \cdot 10^{-2} \nless 0.1 \frac{0.145}{0.4} 0.25 \lesssim 9.1 \cdot 10^{-3}$ & No \\
$\alpha$ & $10^{-3}<0.1 \frac{0.145}{0.9} 0.25 \gtrsim 4 \cdot 10^{-3}$ & Yes
\end{tabular}

be included as an unknown in the inverse problem. All other parameters have to be included as unknowns in the inverse problem.

\section{Simplified Model of Lock-in Image}

To address the noise associated with complex random variables, we introduce the so called circular complex normal distribution, denoted as $C N\left(0, \sigma^{2}\right)$, with probability density function $f(z)=\frac{1}{\pi \sigma^{2}} e^{-\frac{|z|^{2}}{\sigma^{2}}}, \quad \forall z \in \mathbb{C}$, (see [28). A complex random variable $Z=X+i Y$ is $C N\left(0, \sigma^{2}\right)$ if $X$ and $Y$ are independent and identically distributed variables according to the normal distribution $N\left(0, \sigma^{2} / 2\right)$.

We denote the detector plane of an IR camera as $\widetilde{\Pi} \subset \mathbb{R}^{2}$. We define the bijective application $\boldsymbol{x}=P(\widetilde{\boldsymbol{x}})$, with

$$
P: \widetilde{\Pi} \subset \mathbb{R}^{2} \rightarrow \omega \subset \partial \Omega \subset \mathbb{R}^{3},
$$

that relates the coordinates $\widetilde{\boldsymbol{x}}$ of a point at the detector plane with point $\boldsymbol{x}$ at the focused surface $\omega \subset \partial \Omega$. The line segment that joins a point $\boldsymbol{x}$ on the surface of the sample with $\widetilde{\boldsymbol{x}}$ goes through the focal point of the IR camera.

The experimental thermograms obtained from the signal in phase and quadrature contain discrete information. This data can be restructured in one noisy complex vector

${ }_{210}$ of dimension $\widetilde{N}$, where $\widetilde{N}$ is the number of detector cells, $\left\{u_{d, k}\right\}_{k=1}^{\widetilde{N}}$. The in phase and quadrature thermograms correspond to the real and imaginary parts, respectively.

We define the application $\widetilde{\boldsymbol{x}}_{k}=S(k)$ such that

$$
S:\{1, \ldots, \widetilde{N}\} \rightarrow \widetilde{\Pi} \subset \mathbb{R}^{2}
$$

relates the $k$-th pixel of the detector with the central coordinate $\widetilde{\boldsymbol{x}}_{k}$ of the aforementioned pixel.

We also project the numerical approximation $u_{h}$ restricted to a region $\omega$ on the surface of the sample to the detector plane. To do so, we define $\widetilde{u}_{h}: \widetilde{\Pi} \rightarrow \mathbb{C}$ as:

$$
\widetilde{u}_{h}(\widetilde{\boldsymbol{x}})=\int_{\mathbb{R}^{2}} k_{\text {opt }}(\widetilde{\boldsymbol{x}}-\widetilde{\boldsymbol{y}}) u_{h}(P(\widetilde{\boldsymbol{y}})) d \widetilde{\boldsymbol{y}},
$$


where $k_{\text {opt }}$ is the wave dispersion function in the detector plane generated by the optical system. The discretization of $\widetilde{u}_{h}$ conforming to the detector provides the relationship between model and data. For that, we consider the piecewise constant function $w:=$ $P_{0}\left(\widetilde{u}_{h}\right)$, where

$$
P_{0}: L^{2}\left(\mathbb{R}^{2} \rightarrow \mathbb{C}\right) \rightarrow L^{2}\left(\mathbb{R}^{2} \rightarrow \mathbb{C}\right)
$$

is the $L^{2}$ projection of $\widetilde{u}_{h}$ over a piecewise polynomial space of degree zero related to the cells in $\widetilde{\Pi}$.

The assumed model for the complex-valued data of the lock-in thermogram is:

$$
u_{d, k}=z_{0} w_{k}+w_{k} \eta_{m}^{k}+\sqrt{\left|w_{k}\right|} \eta_{p}^{k}+\eta_{a}^{k}, \quad k=1,2, \cdots, \widetilde{N},
$$

where $w_{k}:=w\left(\widetilde{\boldsymbol{x}}_{k}\right), z_{0} \in \mathbb{C}$ is a conversion factor (we select $z_{0}=1$ for simplicity); $\eta_{m}^{k}, \eta_{p}^{k}$ and $\eta_{a}^{k}$ are random variables with normal distribution and null mean. They are associated with a multiplicative noise, a Poisson noise, and an additive noise, respectively. We assume that $\eta_{m}^{k} \sim C N\left(0, \sigma_{m}^{2}\right), \eta_{p}^{k} \sim C N\left(0, \sigma_{p}^{2}\right)$, and $\eta_{a}^{k} \sim C N\left(0, \sigma_{a}^{2}\right)$ are white noises with uncorrelated variables. Thus, the sum of all of them at one pixel is a random variable with circular complex normal distribution:

$$
\eta^{k}\left(u_{d, k}\right)=w_{k} \eta_{m}^{k}+\sqrt{\left|w_{k}\right|} \eta_{p}^{k}+\eta_{a}^{k} \sim C N\left(0, \sigma^{2}\left(w_{k}\right)\right),
$$

where

is the noise variance.

$$
\sigma^{2}\left(w_{k}\right)=\sigma_{m}^{2}\left|w_{k}\right|^{2}+\sigma_{p}^{2}\left|w_{k}\right|+\sigma_{a}^{2}
$$

\section{Weighted Least Squares Fitting}

We want to fit the projected solution $w_{i}$, defined in Section 5, to the experimental thermograms $u_{d, i}$. To do this, we propose the following non-linear weighted least-squares method:

$$
\underset{\beta}{\arg \min } \sum_{i=1}^{N} \frac{\left|u_{d, i}-w_{i}(\beta)\right|^{2}}{\sigma_{m}^{2}\left|w_{i}\right|^{2}+\sigma_{p}^{2}\left|w_{i}\right|+\sigma_{a}^{2}},
$$

where $N$ is the number of pixels. To solve $(15)$, we employ a linearization process at each iteration of the Levenderg-Madquardt optimization algorithm. To start the iterative method, we consider $w_{i} \approx u_{d, i}$. Notice that a proper analysis region should be selected -with signal $u_{d, i}$ away from zero- to avoid null denominators.

\subsection{Classical Fittings and Noise Types}

We illustrate the behavior of the WLS method (15) with three extreme noise-type cases: additive, multiplicative, and Poisson-dominant noise. In these extreme cases, the fitting reduces to a classical case of least-squares fitting. We illustrate these extreme cases in the following:

a) The simplest case occurs when the additive noise is dominant $\left(\sigma_{a} \gg \sigma_{m}\right.$ and $\left.\sigma_{a} \gg \sigma_{p}\right)$. Here, 15 becomes similar to the classical least square fitting

$$
\underset{\beta}{\arg \min } \sum_{i=1}^{N}\left|u_{d, i}-w_{i}(\beta)\right|^{2} .
$$


b) In the case that the multiplicative noise is dominant $\left(\sigma_{m} \gg \sigma_{p}\right.$ and $\left.\sigma_{m} \gg \sigma_{a}\right)$, (15) is similar to minimize

$$
\sum_{i=1}^{N}\left|\log \left(u_{d, i}\right)-\log \left(w_{i}(\beta)\right)\right|^{2}=\sum_{i=1}^{N}\left|\log \left(\frac{u_{d, i}}{w_{i}(\beta)}\right)^{2}\right|
$$

since, assuming $\frac{u_{d, i}}{w_{i}(\beta)}-1 \approx 0$ and applying the approximation $\log (1+x) \approx x$ when $x \rightarrow 0$, 16) becomes

$$
\sum_{i=1}^{N}\left|\frac{u_{d, i}}{w_{i}(\beta)}-1\right|^{2}=\sum_{i=1}^{N} \frac{\left|u_{d, i}-w_{i}(\beta)\right|^{2}}{\left|w_{i}(\beta)\right|^{2}} .
$$

In 23. we solved an inverse problem to determine the thermal resistance $R$ by the minimization of the discrepancy of the phases and the logarithms of the amplitudes. This is equivalent to minimize (16) due to the relation shown in (7). Therefore, the method applied in 23] assumes multiplicative noise as dominant in the measured thermograms. Furthermore, to avoid numerical problems that arise when computing the imaginary part of the principal logarithm, we recommend to implement the minimization of (17) instead of (16), that is:

$$
\underset{\beta}{\arg \min } \sum_{i=1}^{N} \frac{\left|u_{d, i}-w_{i}(\beta)\right|^{2}}{\left|w_{i}(\beta)\right|^{2}} .
$$

c) When Poisson noise is dominant $\left(\sigma_{p} \gg \sigma_{m}\right.$ and $\left.\sigma_{p} \gg \sigma_{m}\right)$ and there is a large signal-to-noise ratio $\sqrt{\left|w_{i}(\beta)\right|} \gg \eta_{p}$, we can approximate 15 by

$$
\underset{\beta}{\arg \min } \sum_{i=1}^{N}\left|\sqrt{\left|u_{d, i}\right|} e^{i \phi_{u_{d, i}}}-\sqrt{\left|w_{i}(\beta)\right|} e^{i \phi_{w_{i}}}\right|^{2} \text {. }
$$

6.2. Theoretical Uncertainty of the Reconstructed Parameters in OLT Least Square Fitting

Let

$$
\boldsymbol{x}^{\eta}=\left(A^{t} A\right)^{-1} A^{t} \boldsymbol{b}^{\delta},
$$

be the least square solution of the approximation $A \boldsymbol{x}^{\eta} \approx \boldsymbol{b}^{\delta}$, where $\boldsymbol{b}^{\delta}=\boldsymbol{b}+\boldsymbol{\delta}$ is the noisy data with each component of $\boldsymbol{\delta}$ being $\delta_{i} \sim N\left(0, \sigma^{2}\right)$. Let $\boldsymbol{x}_{0}=A^{-1} \boldsymbol{b}$. Classical results (see [29], 30]) establish that the least square solution is $\boldsymbol{x}^{\eta}=\boldsymbol{x}_{0}+\boldsymbol{\eta}$, where $\eta_{i} \sim N\left(0, \sigma_{i}^{2}\right)$, has the variances relation:

$$
\sigma_{i}^{2}=\left[\left(A^{t} A\right)^{-1}\right]_{i i} \sigma^{2} .
$$

Equation (21) can be extended to the complex case as

$$
\boldsymbol{x}^{\eta}=\left(A^{\mathrm{H}} A\right)^{-1} A^{\mathrm{H}} \boldsymbol{b}^{\delta},
$$


where $A^{\mathrm{H}}$ denotes the Hermitian matrix of $A, \delta_{i} \sim C N\left(0, \sigma^{2}\right), \eta_{i} \sim C N\left(0, \sigma_{i}^{2}\right)$. The variances relation becomes

$$
\sigma_{i}^{2}=\left[\left(A^{\mathrm{H}} A\right)^{-1}\right]_{i i} \sigma^{2} .
$$

In our case, we have real parameters $\boldsymbol{x}^{\eta}$ and complex data $\boldsymbol{b}^{\delta}$. Thus, neither the least-squares real solution given by 20 nor the complex-valued one 222 are applicable to our case. To resolve this issue, we propose a least-squares method, whose solution is

$$
\boldsymbol{x}^{\eta}=\left(\Re\left(A^{\mathrm{H}} A\right)\right)^{-1} \Re\left(A^{\mathrm{H}} \boldsymbol{b}^{\delta}\right),
$$

assuming $\delta_{i} \sim C N\left(0, \sigma^{2}\right)$ and $\eta_{i} \sim N\left(0, \sigma_{i}^{2}\right)$, with the variances relation

$$
\sigma_{i}^{2}=\frac{1}{2}\left[\left(\Re\left(A^{\mathrm{H}} A\right)\right)^{-1}\right]_{i i} \sigma^{2} .
$$

\subsection{Influence of the Dataset Selection in the Precision of Micro-crack Thickness Esti- mation}

It is desirable to consider the smallest possible dataset from the experimental thermograms that at the same time incorporates relevant information about all the involved parameters.

To analyze the influence that the dataset selection has in the parameters estimation we apply (24), being

$$
\boldsymbol{b}^{\delta}=\left(\begin{array}{c}
\frac{\sigma_{m}}{\sigma\left(w_{1}\right)}\left(u_{d, 1}-w_{1}\right) \\
\vdots \\
\frac{\sigma_{m}}{\sigma\left(w_{N}\right)}\left(u_{d, N}-w_{N}\right)
\end{array}\right)=\left(\begin{array}{c}
\frac{\sigma_{m}}{\sigma\left(w_{1}\right)} \eta\left(u_{d, 1}\right) \\
\vdots \\
\frac{\sigma_{m}}{\sigma\left(w_{N}\right)} \eta\left(u_{d, N}\right)
\end{array}\right)
$$

the weighted residual. An uncertainty quantification is obtained from 25) defining $A=$ $W J D$, where

$$
W=\operatorname{diag}\left(\frac{\sigma_{m}}{\sigma\left(w_{1}\right)}, \cdots, \frac{\sigma_{m}}{\sigma\left(w_{N}\right)}\right)
$$

is the weighting matrix,

$$
J=\left(\begin{array}{cccc}
\frac{\partial w_{1}}{\partial R} & \frac{\partial w_{1}}{\partial a} & \frac{\partial w_{1}}{\partial x_{c 1}} & \frac{\partial w_{1}}{\partial x_{c 2}} \\
\vdots & \vdots & \vdots & \vdots \\
\frac{\partial w_{N}}{\partial R} & \frac{\partial w_{N}}{\partial a} & \frac{\partial w_{N}}{\partial x_{c 1}} & \frac{\partial w_{N}}{\partial x_{c 2}}
\end{array}\right)
$$

and $D=\operatorname{diag}\left(R, a,\left\|\boldsymbol{x}_{c}\right\|\left|,\left\|\boldsymbol{x}_{c}\right\|\right|\right)$ is a diagonal scaling matrix.

To determine uncertainty values, we assume the relative influence $\sigma_{m}=2 \sigma_{p}=10 \sigma_{a}$ between the three types of noise in the data.

Table 2 summarizes the information of six datasets related to the upper surface in Figure 2, $\mathrm{A}$ is the set of complex-valued data of the entire thermogram. B and $\mathrm{C}$ are the dataset inside a rectangle limited by the points showed in Table 2 D, E, and F are the datasets in the segment defined by the two points described in the table. 
Table 2: Information about six data subsets

\begin{tabular}{ccc}
\hline Notation & Points & \# pixels $(N)$ \\
\hline A & all data & 142129 \\
B & $(-2,-1),(2,3)$ & 17822 \\
C & $(-1,-1),(1,2)$ & 6700 \\
D & $(-0.3,-2),(-0.3,2)$ & 133 \\
E & $(0,-2),(0,2)$ & 133 \\
F & $(0.3,-2),(0.3,2)$ & 133
\end{tabular}

Table 3 shows the ratio between the theoretical uncertainty of the recovered parameters $\eta(\beta):=\left(\frac{\Delta R}{R}, \frac{\Delta a}{a}, \frac{\Delta x_{c 1}}{\left\|\boldsymbol{x}_{c}\right\|}, \frac{\Delta x_{c 2}}{\left\|\boldsymbol{x}_{c}\right\|}\right)$ for five sets of $N$ data in the solution and the uncertainty of the data. We obtain subpixel resolution in the estimation of the thermal resistance $R=10^{-4} \mathrm{~m}^{2} \mathrm{~K} \mathrm{~W}^{-1}$, equivalent to a crack thickness of $2.4 \mu \mathrm{m}$, as well as in the rest of the parameters. The relative error in the estimation of $R$ has the same order as the relative error of the multiplicative noise. Also, the use of additional pixels provides higher accuracy, being the most efficient case the use of dataset $\mathrm{C}$. In there, the relative error in the estimation of $R$ is close to the relative error of the multiplicative noise, and it is unnecessary to store a large amount of data. We advise against the use of data contained in one profile (dataset E) to reconstruct the parameters. This dataset amplifies the uncertainty of the center location along the $x_{1}$ axis. It also augments the increase in the uncertainty when determining $R$.

Table 3: Summary of the ratio between standard deviations of relative errors in the parameter estimations and the standard deviations of the multiplicative noise.

\begin{tabular}{|c|c|c|c|c|c|}
\hline Dataset & \# pixels & $\frac{\sigma_{\frac{\Delta R}{R}}}{\sigma_{m}}$ & $\frac{\sigma_{\frac{\Delta a}{a}}}{\sigma_{m}}$ & $\frac{\sigma_{\frac{\Delta x_{c 1}}{\left\|\boldsymbol{x}_{c}\right\|}}}{\sigma_{m}}$ & $\frac{\sigma_{\frac{\Delta x_{c 2}}{\left\|\boldsymbol{x}_{c}\right\|}}}{\sigma_{m}}$ \\
\hline $\mathrm{A}$ & 142129 & 1.155 & 0.050 & 0.012 & 0.012 \\
\hline B & 17822 & 1.179 & 0.052 & 0.012 & 0.012 \\
\hline $\mathrm{C}$ & 6700 & 1.185 & 0.066 & 0.016 & 0.012 \\
\hline $\mathrm{D} \cup \mathrm{E} \cup \mathrm{F}$ & 399 & 3.850 & 0.233 & 0.120 & 0.066 \\
\hline $\mathrm{E}$ & 133 & 5.712 & 0.340 & 90.78 & 0.108 \\
\hline
\end{tabular}

\section{Conclusions}

In OLT problems involving thin cracks (less than $50 \mu \mathrm{m}$ in width), a precision above the pixel resolution may be required for determining certain experimental parameters such as the center location and radius of the laser spot. Such high resolution could be achieved by including them as unknowns and fitting them with a WLS method. We have designed a sensitivity based calibration method to determine the parameters that need to be included in the inverse problem to characterize the thermal resistance with the desired precision. The selection of the dataset to make the reconstruction of the parameters is crucial. It is necessary to develop the procedures to estimate the variances of the noise data associated with the experimental thermograms. Including this information through 
weights in the least square fitting allows obtaining more accurate reconstructions. The above steps are needed to obtain relative accuracies of the crack thickness that are one order of magnitude less than that of the pixel size. As future work, we shall analyze the relative error of the thermal diffusivity. In most practical applications, the thermal diffusivity could be considered as an additional unknown.

\section{Acknowledgements}

This research is supported by the European Union's Horizon 2020 research and innovation program under the Marie Sklodowska-Curie grant agreement No 777778 (MATHROCKS), the European POCTEFA 2014-2020 Project PIXIL (EFA362/19) by the European Regional Development Fund (ERDF) through the Interreg V-A Spain-FranceAndorra program, the Project of the Spanish Ministry of Science and Innovation with reference PID2019-108111RB-I00 (FEDER/AEI), the BCAM "Severo Ochoa" accredi275 tation of excellence (SEV-2017-0718), and the Basque Government through the BERC 2018-2021 program, the two Elkartek projects 3KIA (KK-2020/00049) and MATHEO (KK-2019-00085), the grant "Artificial Intelligence in BCAM number EXP. 2019/00432", and the Consolidated Research Group MATHMODE (IT1294-19) given by the Department of Education. Ricardo Celorrio has received funding from the Spanish project

${ }_{280}$ FEDER/MCYT MTM2016-75139-R and the Diputación General de Aragón (reference group APEDIF, ref. E24-17R), and the Project of the Spanish Ministry of Science and Innovation with reference PID2019-104347RB-I00 (FEDER/AEI).

\section{References}

[1] W. Datong, B. Gerd, Lock-in thermography for nondestructive evaluation of materials, Revue Générale de Thermique 37 (8) (1998) 693 - 703. doi:http://dx.doi.org/10.1016/S0035-3159(98) 80047-0

URL http://www.sciencedirect.com/science/article/pii/S0035315998800470

[2] A. Mendioroz, R. Celorrio, A. Salazar, Ultrasound excited thermography: an efficient tool for the characterization of vertical cracks, Measurement Science and Technology 28 (11) (2017) 112001. doi:10.1088/1361-6501/aa825a URL https://doi.org/10.1088\%2F1361-6501\%2Faa825a

[3] D. Balageas, X. Maldague, D. Burleigh, V. P. Vavilov, B. Oswald-Tranta, J.-M. Roche, C. Pradere, G. M. Carlomagno, Thermal (IR) and other NDT techniques for improved material inspection Journal of Nondestructive Evaluation 35 (1) (2016) 18. doi:10.1007/s10921-015-0331-7

295 URL https://doi.org/10.1007/s10921-015-0331-7

n [4] P. Bison, M. Ceseri, G. Inglese, Comparing laplace and helmholtz approaches to identify the rear-surface profile of a plate by means of periodic thermal data Journal of Modern Optics

57 (18) (2010) 1752-1758. arXiv:https://doi.org/10.1080/09500340.2010.496537, doi:10.1080/ 09500340.2010 .496537

$300 \quad$ URL https://doi.org/10.1080/09500340.2010.496537

[5] S. Quek, D. Almond, L. Nelson, T. Barden, A novel and robust thermal wave signal reconstruction technique for defect detection in lock-in thermography Measurement Science and Technology 16 (5) (2005) 1223. URL http://stacks . iop.org/0957-0233/16/i=5/a=024

305

[6] R. Li Voti, G. L. Liakhou, S. Paoloni, C. Sibilia, M. Bertolotti, Thermal waves physics, Journal of Optoelectronics and Advanced Materials 3 (4) (2001) 779-816.

[7] O. Breitenstein, W. Warta, M. Langenkamp, Lock-in Thermography, Springer Series in Advanced Microelectronics, Springer-Verlag Berlin Heidelberg, 2010. 
[8] J. Rantala, D. Wu, G. Busse, Amplitude-modulated lock-in vibrothermography for nde of polymers 310 and composites Research in Nondestructive Evaluation 7 (4) (1996) 215-228. arXiv: https://www. tandfonline.com/doi/pdf/10.1080/09349849609409580 doi:10.1080/09349849609409580

URL https ://www . tandfonline . com/doi/abs/10.1080/09349849609409580

[9] A. Mendioroz, A. Castelo, R. Celorrio, A. Salazar, Characterization of vertical buried defects using lock-in vibrothermography: I. direct problem, Measurement Science and Technology 24 (6) (2013) 065601 .

URL http://stacks .iop.org/0957-0233/24/i=6/a=065601

[10] R. Celorrio, A. Mendioroz, A. Salazar, Characterization of vertical buried defects using lock-in vibrothermography: II. inverse problem Measurement Science and Technology 24 (6) (2013) 065602. URL http://stacks . iop. org/0957-0233/24/i=6/a=065602

320 [11] G. Riegert, T. Zweschper, G. Busse, Lockin thermography with eddy current excitation, Quantita-

1) tive InfraRed Thermography Journal 1 (1) (2004) 21-32. arXiv:https://doi.org/10.3166/qirt. 1.21-32 doi:10.3166/qirt.1.21-32

URL https://doi.org/10.3166/qirt.1.21-32

325

[12] N. W. Pech-May, A. Oleaga, A. Mendioroz, A. J. Omella, R. Celorrio, A. Salazar, Vertical cracks characterization using lock-in thermography: I infinite cracks, Measurement Science and Technology 25 (11) (2014) 115601. doi:10.1088/0957-0233/25/11/115601 URL https : //doi.org/10.1088\%2F0957-0233\%2F $25 \% 2$ F $11 \% 2$ F 115601

[13] D. Wu, G. Busse, Lock-in thermography for nondestructive evaluation of materials Revue Générale de Thermique 37 (8) (1998) 693 - 703. doi :http://dx.doi.org/10.1016/S0035-3159(98)80047-0 URL http://www.sciencedirect.com/science/article/pii/S0035315998800470

[14] J. Schlichting, C. Maierhofer, M. Kreutzbruck, Crack sizing by laser excited thermography, NDT \& E International 45 (1) (2012) 133 - 140. doi:http://dx.doi.org/10.1016/j.ndteint.2011.09.014 URL http://www.sciencedirect.com/science/article/pii/S0963869511001502

[15] S. Beuve, Z. Qin, J.-P. Roger, S. Holé, C. Boué, Open cracks depth sizing by multi-frequency laser stimulated lock-in thermography combined with image processing, Sensors and Actuators A: Physical 247 (2016) 494 - 503. doi:https://doi.org/10.1016/j.sna.2016.06.028 URL http://www.sciencedirect.com/science/article/pii/S0924424716303144

[16] C. Boué, S. Holé, Open crack depth sizing by multi-speed continuous laser stimulated lock-in ther-

1. mography, Measurement Science and Technology 28 (6) (2017) 065901. doi:10.1088/1361-6501/ 340 aa687b URL https://doi.org/10.1088\%2F1361-6501\%2Faa687b

[17] R. Celorrio, A. Omella, A. Mendioroz, A. Oleaga, A. Salazar, Advances in crack characterization by lock-in infrared thermography International Journal of Thermophysics 36 (5) (2015) 1202-1207. doi:10.1007/s10765-014-1676-3 URL http://dx.doi.org/10.1007/s10765-014-1676-3

[18] P. Magnan, Detection of visible photons in CCD and CMOS: A comparative view, Nuclear Instruments and Methods in Physics Research Section A: Accelerators, Spectrometers, Detectors and Associated Equipment 504 (1-3) (2003) 199 - 212, proceedings of the 3rd International Conference on New Developments in Photodetection. doi:http://dx.doi.org/10.1016/S0168-9002(03)00792-7

350 URL http://www.sciencedirect.com/science/article/pii/S0168900203007927 video cameras, Measurement Science and Technology 19 (4) (2008) 045207.

URL http://stacks.iop.org/0957-0233/19/i=4/a=045207

[20] S. Hasinoff, Photon, Poisson Noise Springer US, Boston, MA, 2014, pp. 608-610. doi:10.1007/ 978-0-387-31439-6_482

URL http://dx.doi.org/10.1007/978-0-387-31439-6_482

[21] M. Streza, Y. Fedala, J. Roger, G. Tessier, B. Boue, Heat transfer modeling for surface crack depth evaluation Measurement Science and Technology 24 (4) (2013) 045602.

URL http://stacks . iop. org/0957-0233/24/i=4/a=045602

360 [22] A. Omella, R. Celorrio, Discontinuous Galerkin method applied to photothermal inspection of cracked materials, Vol. 40 of Monografías Matemáticas García de Galdeano, Prensas de la Universidad de Zaragoza, 2016, pp. 105-114.

[23] R. Celorrio, A. J. Omella, N. W. Pech-May, A. Oleaga, A. Mendioroz, A. Salazar, Vertical cracks characterization using lock-in thermography: II finite cracks. Measurement Science and Technology 25 (11) (2014) 115602. doi:10.1088/0957-0233/25/11/115602

URL https://doi.org/10.1088\%2F0957-0233\%2F $25 \% 2 \mathrm{~F} 11 \% 2 \mathrm{~F} 115602$

[24] A. Romkes, S. Prudhomme, J. Oden, A priori error analyses of a stabilized discontinuous galerkin 
method. Computers \& Mathematics with Applications 46 (8) (2003) 1289 - 1311. doi:https: //doi.org/10.1016/S0898-1221(03)90220-3.

370 URL http://www.sciencedirect.com/science/article/pii/S0898122103902203

[25] A. Logg, K.-A. Mardal, G. Wells (Eds.), Automated Solution of Differential Equations by the Finite Element Method. Vol. 84 of Lecture Notes in Computational Science and Engineering, Springer, 2012. doi:10.1007/978-3-642-23099-8

URL http://dx.doi.org/10.1007/978-3-642-23099-8

$375[26]$ J. Fivez, J. Thoen, On the accuracy of depth-dependent thermal conductivity retrieval in photoa-

coustic experiments, Journal of Applied Physics 81 (7) (1997) 2963-2965. arXiv:http://dx.doi. org/10.1063/1.364328 doi:10.1063/1.364328

URL http://dx.doi .org/10.1063/1.364328

[27] F. Cernuschi, P. G. Bison, A. Figari, S. Marinetti, E. Grinzato, Thermal diffusivity measurements by photothermal and thermographic techniques, International Journal of Thermophysics 25 (2) (2004) 439-457. doi:10.1023/B:IJOT.0000028480.27206.cb

URL https://doi.org/10.1023/B:IJOT.0000028480.27206.cb

[28] E. Ollila, J. Eriksson, V. Koivunen, Complex elliptically symmetric random variables-generation, characterization, and circularity tests, IEEE Transactions on Signal Procesing 59 (1) (2011) 58-69.

[29] J. Bagilvo, 14. Linear Least Squares Analysis ASA-SIAM, 2005, Ch. 14, pp. 205226. arXiv:http://epubs.siam.org/doi/pdf/10.1137/1.9780898718416.ch14 doi:10.1137/1. 9780898718416.ch14

URL http://epubs.siam.org/doi/abs/10.1137/1.9780898718416.ch14

[30] P. Richter, Estimating errors in least-squares fitting, Tech. Rep. TDA Progress Report 42-122, NASA (08 1995). 